\title{
PROFISSIONAIS DE SAÚDE MENTAL: ESTRESSE E ESTRESSORES OCUPACIONAIS STRESS E ESTRESSORES OCUPACIONAIS EM SAÚDE MENTAL
}

\author{
Ana Flávia de Oliveira Santos* \\ Carmen Lúcia Cardoso
}

\begin{abstract}
RESUMO. Os profissionais de saúde mental se encontram particularmente vulneráveis ao estresse, em vista das características do trabalho que desempenham. O objetivo do estudo foi avaliar manifestações de estresse, autopercepção de estresse e fatores estressantes do trabalho em profissionais de serviços substitutivos de saúde mental. Participaram 25 trabalhadores com pelo menos seis meses de atuação profissional. Aplicaram-se os instrumentos Inventário de Sintomas de Estresse para Adultos de Lipp e Roteiro Complementar. Os dados quantitativos foram tratados por meio de estatística descritiva, e os demais, analisados qualitativamente. Verificou-se que $36,0 \%$ dos profissionais apresentavam manifestação de estresse e que 44,0\% deles se percebiam sob estresse. Condições de trabalho e relacionamento no trabalho foram os fatores mais frequentemente associados à percepção de estar sob estresse. Concluiu-se que os profissionais vivenciam estressores associados às mudanças relativas ao paradigma psicossocial de atenção, o que mostra a necessidade de intervenção direcionada ao desenvolvimento de estratégias de enfrentamento às situações ocupacionais estressoras.
\end{abstract}

Palavras-chave: Estresse; estresse ocupacional; trabalhadores.

\section{MENTAL HEALTH PROFESSIONALS: STRESS AND OCCUPATIONAL STRESSORS STRESS AND OCCUPATIONAL STRESSORS OF MENTAL HEALTH}

\begin{abstract}
Mental health professionals are particularly vulnerable to stress,_considering the characteristics of the work they develop. The purpose of this study was to assess the manifestation of stress, self-perception of stress, and stressing work factors in substitutive mental health service professionals. Twenty-five workers, working for at least six months, took part in the study. The instruments employed were Lipp's Inventory of Stress Symptoms in Adults and a Complementary Script. Quantitative data were treated by descriptive statistics, and the remaining data were analyzed qualitatively. It was observed that $36.0 \%$ of the professional had stress manifestations, and $44.0 \%$ perceived they were under stress. Work conditions and relationships at work were the most frequent factors associated to the perception of being under stress. In conclusion, professionals experience stressors associated with the changes implied in the psychosocial paradigm of care. Thus, there is a need to make direct interventions toward the development of coping strategies in stressing occupational situations.
\end{abstract}

Key words: Stress; burnout; workers.

\section{PROFESIONALES DE LA SALUD MENTAL: ESTRÉS Y ESTRESORES OCUPACIONALESESTRÉS Y ESTRESORES OCUPACIONALES EN SALUD MENTAL}

RESUMEN. Los profesionales de la salud mental se encuentran particularmente vulnerables al estrés, teniendo en vista las características del trabajo que desempeñan. El objetivo del estudio fue evaluar manifestación de estrés, auto percepción de estrés y factores estresantes del trabajo en profesionales de servicios sustitutivos de salud mental. Participaron 25 trabajadores actuantes hace por lo menos seis meses. Se aplicaron los instrumentos Inventario de Síntomas de Estrés para Adultos de Lipp y Guión Complementario. Los datos cuantitativos fueron tratados por medio de estadística descriptiva y los demás, analizados cualitativamente. Se verificaron 36,0\% de los profesionales con manifestación de estrés y 44,0\% percibiéndose bajo estrés. Condiciones de trabajo y relación en el trabajo fueron los factores más frecuentemente asociados a la percepción de estar bajo estrés. Se concluye que los profesionales vivencian estresores asociados a los cambios relativos al paradigma psicosocial de atención, apuntando a la necesidad de intervención dirigida al desarrollo de estrategias de enfrentamiento de las situaciones ocupacionales estresoras.

Palabras-clave: Estrés; agotamiento profesional; trabajadores.

Psicóloga e Mestranda pela Faculdade de Filosofia, Ciências e Letras de Ribeirão Preto da Universidade de São Paulo-USP, Brasil.

\# Doutora em Psicologia e Saúde. Professora do Programa de Pós-Graduação em Psicologia do Departamento de Psicologia e Educação da Faculdade de Filosofia, Ciências e Letras de Ribeirão Preto da Universidade de São Paulo, Brasil. 
Selye (1956) foi o primeiro autor a utilizar na área da saúde o termo estresse, caracterizando-o como uma "Síndrome Geral de Adaptação" (SGA) decorrente de um evento que exige esforço do indivíduo em termos de adaptação. Este estressor gera a quebra da homeostase interna do indivíduo, alterando a capacidade do organismo de manter sua constância.

Para representar o processo de estresse, Selye (1956) apresentou um modelo trifásico, constituído por: a) fase de alerta: reação de alerta preparando o indivíduo para a luta ou a fuga através da ativação de mecanismos homeostáticos; b) fase de resistência: quando o organismo tenta restabelecer o equilíbrio interno através de uma ação reparadora, gastando energia para esta adaptação; e c) fase de exaustão: caracterizada pela exaustão física e psicológica, já que a adaptabilidade do organismo ou energia adaptativa é finita, momento em que as doenças se manifestam, podendo ser fatais (Lipp \& Malagris, 1998). Recentemente, em estudo desenvolvido por Lipp (2000), identificou-se uma quarta fase entre a resistência e a exaustão, denominada quase exaustão, momento em que há um enfraquecimento do indivíduo, que, ao não conseguir se adaptar ou resistir ao estressor, torna-se vulnerável ao aparecimento de doenças.

Nos últimos anos, estudos sobre estresse foram conduzidos em múltiplos contextos, associando-o a diferentes variáveis. Entre esses estudos, tem-se observado a preocupação com a investigação acerca da relação entre estresse e trabalho, destacando-se o construto do estresse ocupacional.

A definição mais abrangente neste campo descreve o estresse ocupacional como um processo estressor-resposta, enfatizando em conjunto tanto os fatores do trabalho que excedem a capacidade de enfrentamento do indivíduo (estressores organizacionais) quanto as respostas fisiológicas, psicológicas e comportamentais aos eventos avaliados como estressores (Paschoal \& Tamayo, 2004). Esta abordagem atribui importância à percepção pessoal como mediadora do impacto do estressor no indivíduo, uma vez que, conforme pontuam Lazarus e Folkman (1984), é a percepção e a interpretação da situação como estressora pelo indivíduo o que confere ao estímulo tal potencial e capacidade.

Neste sentido, o estresse ocupacional é definido como a soma de respostas físicas e mentais, bem como reações fisiológicas que, quando intensificadas de modo a exceder a capacidade de enfrentamento do indivíduo, transformam-se em reações emocionais negativas (Yarker, Donaldson-Feilder \& Flaxman, 2007).
Em revisão da literatura, Paschoal e Tamayo (2004) apontam que os estressores organizacionais podem ser de natureza física (ventilação, iluminação, etc.) ou psicossociais (fatores intrínsecos ao trabalho, aspectos do relacionamento interpessoal, autonomia ou controle no trabalho, estressores baseados nos papéis - conflito e ambiguidade de papéis - e fatores relacionados ao desenvolvimento de carreira). $\mathrm{Na}$ área do estresse ocupacional, o modelo demanda-controle de Karasek tem se tornado referência, propondo que a partir de duas dimensões psicossociais - controle no trabalho e demanda psicológica oriunda do trabalho identificam-se situações ocupacionais que causam impacto na saúde do indivíduo (Araújo, Graça \& Araújo, 2003).

As respostas psicológicas mais frequentemente associadas ao estresse ocupacional são ansiedade, insatisfação e depressão (Paschoal \& Tamayo, 2004). $\mathrm{O}$ estresse ocupacional apresenta impacto negativo na saúde dos trabalhadores, bem como no funcionamento das organizações, ocasionando perda de produtividade, diminuição da qualidade dos produtos e dos serviços prestados (Yarker et al., 2007).

Como reação ao estresse ocupacional crônico, os trabalhadores podem desenvolver o burnout, uma síndrome psicológica decorrente de uma má adaptação a um trabalho estressante, prolongado e com elevada carga tensional. O burnout possui três dimensões: esgotamento emocional, despersonalização e redução da realização pessoal (Maslach, Schaufeli \& Leiter, 2001).

A síndrome de burnout apresenta consequências tanto para a organização, quanto para a saúde do trabalhador. Entre as primeiras se situam a força de trabalho alijada de sua inovação, dedicação, criatividade e produtividade (Maslach \& Leiter, 1999). Em relação ao impacto na saúde, os trabalhadores podem apresentar indicadores de estresse ou alguma disfunção da saúde mental, como ansiedade e queda da autoestima, juntamente com diminuída capacidade de enfrentar estressores crônicos (Maslach et al., 2001).

Entre as profissões que demandam intenso contato interpessoal destacam-se as relacionadas à saúde e, mais especificamente, à saúde mental. Tais profissionais se encontram cotidianamente em intenso contato com usuários com dificuldades emocionais, oferecendo-lhes atenção e cuidado às suas necessidades. Carson e Fagin (1996) ressaltam que o estresse e o burnout afetam o cuidado oferecido ao paciente, o nível de desempenho profissional, a satisfação em relação ao trabalho e a própria saúde destes profissionais. 
Por sua complexidade e especificidade, o trabalho em saúde mental é considerado de acentuada vulnerabilidade à sobrecarga emocional. Entre os estressores ocupacionais identificados por profissionais de saúde mental figuram o trato com pessoas que sofrem de transtornos mentais severos, sobrecarga de trabalho, falta de pessoal e conflitos com colegas (Burrows \& McGrath, 2000; Macedo, 2005).

Swoboda et al. (2006) encontraram que a ambiguidade de função, conflitos na equipe, a doença do usuário e a falta de tempo constituem aspectos do trabalho vivenciados como estressantes, ao passo que o contato com usuários e com a equipe foram percebidos como recompensadores. Silva e Costa (2008), em estudo atual considerando o paradigma do modelo psicossocial de atenção, evidenciaram indicadores de sofrimento psíquico entre os profissionais, envolvendo dificuldades nas relações em equipe, com o usuário e com a organização.

Neste sentido, o presente estudo visa identificar e avaliar a manifestação de estresse, a autopercepção quanto ao estresse e a percepção de fatores estressantes no trabalho em profissionais de saúde mental.

\section{MÉTODO}

\section{Participantes}

O presente trabalho foi realizado com 25 trabalhadores de saúde mental de serviços ambulatoriais e de internação parcial de saúde mental de uma cidade do Interior do Estado de São Paulo, número que equivale a $73,5 \%$ do total de trabalhadores de serviços substitutivos da referida cidade. Tais serviços se encontram organizados segundo a Reforma Psiquiátrica e consistem em dispositivos comunitários de atenção em saúde mental caracterizados pelo modo de intervenção psicossocial.

Para participar deste estudo foram convidados a colaborar todos os trabalhadores de saúde mental dos referidos serviços. Estabeleceram-se como critério de inclusão ser trabalhador de saúde mental contratado pelos serviços pesquisados e estar empregado no serviço há, pelo menos, seis meses.

A amostra foi caracterizada, em sua maioria, por profissionais do sexo feminino $(n=19)$ e, em relação à idade, predominou profissionais com mais de 40 anos $(\mathrm{n}=16)$. Dentre os participantes da pesquisa, 12 possuem o ensino superior, seguido de seis que realizaram pós-graduação. Quanto ao estado civil, 13 são casados. Entre os participantes, 21 possuem religião, dos quais 14 relatam ser praticantes na religião.

\section{Instrumentos}

Para a coleta de dados foram utilizados o Inventário de Sintomas de Estresse para Adultos de Lipp - ISSL e um roteiro complementar elaborado pela pesquisadora.

O Inventário de Sintomas de Estresse para Adultos, validado por Lipp (2000), permite avaliar tanto o estado de estresse do respondente, a partir da identificação da presença de sintomas característicos, quanto a fase do estresse e o tipo de sintomatologia (se somática ou psicológica). O ISSL, composto por 53 itens divididos em três partes, avalia, primeiramente, os sintomas físicos e psicológicos das últimas 24 horas (15 itens); posteriormente, os sintomas manifestados na última semana (15 itens) e, por fim, os sintomas experimentados no último mês ( 23 itens);

$\mathrm{O}$ roteiro complementar elaborado pela pesquisadora baseia-se em estudos nos quais se avaliaram fatores estressantes no trabalho de profissionais da saúde (Burrows \& McGrath, 2000; Macedo, 2005), que visa identificar:

a) aspectos sociodemográficos dos trabalhadores (6 itens);

b) percepção de fatores estressantes: de acordo com uma escala do tipo Likert de 5 pontos de intensidade variando de "nada estressante" a "muito estressante", dos seguintes aspectos do trabalho: "o trabalho", "trabalhar em equipe", "trabalhar diretamente com pessoas", "contato com os usuários atendidos", "contato com os colegas de trabalho" e "ambiente e condições de trabalho";

c) autopercepção do estresse: composto de uma questão fechada relativa à percepção do próprio estresse segundo uma escala tipo Likert de 5 pontos de intensidade, variando de "nada estressado" a "muito estressado". No caso de se perceberem com algum grau de estresse, deveriam responder a uma questão aberta acerca dos fatores que consideravam estar associados ao estresse percebido.

\section{Procedimentos de coleta e análise de dados}

O presente trabalho foi aprovado pelo Comitê de Ética em Pesquisa, mediante o protocolo $\mathrm{n}^{\circ}$ 0167/CEP/CSE-FMRP-USP. Inicialmente, foi ressaltado o caráter voluntário da colaboração, obtendo-se o consentimento do profissional em participar do estudo e sua assinatura do termo de Consentimento Livre e Esclarecido. Em seguida, em aplicação individual, procedeu-se à autoadministração do roteiro complementar respondido na presença da pesquisadora, que esteve disponível para eventuais 
esclarecimentos, a que se seguiu a administração do ISSL.

As questões fechadas do roteiro complementar quanto aos fatores estressantes do trabalho e à autopercepção do estresse foram organizadas e tratadas por meio de estatística descritiva. Os dados do ISSL foram cotados segundo suas proposições técnicas e tratados por meio de estatística descritiva.

Os dados referentes à questão aberta quanto à autopercepção do estresse foram analisados segundo as proposições da Análise de Conteúdo (Bardin, 1979). Após uma leitura flutuante das respostas dos participantes, procedeu-se à sua categorização, considerando-se a frequência das respostas.

\section{RESULTADOS}

A partir dos dados referentes ao ISSL, verificouse que $36,0 \%(n=9)$ dos participantes apresentaram estresse, e destes, $88,9 \%(n=8)$ encontravam-se na fase de resistência e $11,1 \%$ na de exaustão $(n=1)$. A sintomatologia apresentada foi predominantemente física $(66,7 \%$ - n=6). Quanto à avaliação individual e subjetiva do estresse, $44,0 \%(n=11)$ se perceberam como muito estressados e $32,0 \%(n=8)$ como pouco estressados.

A tabela 1 apresenta os resultados descritivos relativos ao roteiro complementar quanto à percepção do estresse em relação aos fatores do trabalho.

Tabela 1. Avaliação Quanto à Percepção dos Fatores de Estresse no Trabalho em Saúde Mental.

\begin{tabular}{lcc}
\hline \multicolumn{1}{c}{ Fatores } & Estressante & n \\
\hline \multirow{2}{*}{ Trabalho } & Muito & 15 \\
& Moderado & 8 \\
& Pouco & 2 \\
\hline \multirow{2}{*}{ Trabalhar em equipe } & Muito & 10 \\
& Moderado & 6 \\
& Pouco & 9 \\
\hline \multirow{3}{*}{ Trabalhar diretamente com pessoas } & Muito & 10 \\
& Moderado & 5 \\
& Pouco & 10 \\
\hline \multirow{3}{*}{ Contato com usuários atendidos } & Muito & 9 \\
& Moderado & 5 \\
& Pouco & 11 \\
\hline \multirow{2}{*}{ Contato com colegas de trabalho } & Muito & 8 \\
& Moderado & 5 \\
& Pouco & 12 \\
\hline \multirow{2}{*}{ Ambiente e condições de trabalho } & Muito & 12 \\
& Moderado & 4 \\
& Pouco & 9 \\
\hline
\end{tabular}

A categorização dos dados da questão aberta do Roteiro Complementar possibilitou verificar que os participantes que informaram perceberem-se com algum nível de estresse $(n=23)$ atribuíram-no a fatores ligados ao trabalho $(n=23)$ ou a fatores de ordem pessoal $(n=2)$. A análise das respostas fornecidas permitiu a formulação de categorias que descrevem os fatores atrelados às manifestações de estresse, as quais são apresentadas a seguir, no quadro 1, em ordem decrescente de frequência de respostas.

\begin{tabular}{|cl|}
\hline Categoria & \multicolumn{1}{c|}{ Subcategorias } \\
\hline & Sobrecarga de trabalho \\
& - Falta de profissionais; \\
& - Demanda pelo serviço. \\
$\begin{array}{c}\text { Condições de } \\
\text { trabalho }\end{array}$ & Gestão do trabalho \\
& - Estrutura física precária; \\
& - Dificuldades no gerenciamento administrativo; \\
& - Falta de suporte ao profissional; \\
& - Palta de recursos materiais; \\
& - Dessaro no trabalho; \\
& Dificuldades na execução do trabalho em equipe \\
& - Falta de confiança nos colegas de trabalho; \\
& - Diferença de valores na equipe; \\
Relacionamento & - Atribuição de valores negativos; \\
no trabalho & - Consideração depreciativa da conduta do colega. \\
& Dificuldades no relacionamento interpessoal \\
& - Dificuldades com profissionais; \\
& - Dificuldades com usuários. \\
\hline & - Baixa remuneração; \\
Falta de & - Frustração quanto aos resultados do trabalho; \\
recompensa no & - Falta de reconhecimento; \\
trabalho & - Baixa qualidade do cuidado. \\
\hline Vida pessoal & - Problemas familiares. \\
\hline
\end{tabular}

Quadro 1: Fatores Estressantes Categorizados a Partir das Respostas dos Participantes que se Percebem Sob Estresse.

\section{DISCUSSÃO}

Um maior percentual indica que os trabalhadores se percebem como muito estressados $(n=11)$ quando comparados com os dados de manifestação de estresse obtidos pelo ISSL $(n=9)$. Tendo em vista que, em geral, a palavra estresse é empregada associada a sensações de desconforto e a consequências negativas, sendo crescente o número de pessoas que se avaliam como estressadas (Stacciarini \& Troccolo, 2001), pode-se supor que os profissionais vivenciem dificuldades que nomeiam como estresse. Uma vez que tais resultados apontam para uma mesma direção, pode-se inferir que os profissionais de saúde mental deste estudo vivenciam manifestações de estresse. 
A maioria dos participantes que apresentam estresse, de acordo com o ISSL, encontra-se na fase de resistência $(88,9 \%)$, momento em que o estresse já se encontra instalado, demandando do indivíduo dispêndio de energia na tentativa de restabelecer o equilíbrio interno por meio de ação reparadora, o que pode levar ao enfraquecimento físico do indivíduo e, caso passe para fases mais avançadas do estresse, acarreta o risco de desenvolver doenças. Tendo em vista que o processo de enfrentamento de uma situação estressora se inicia com a percepção e avaliação do estressor como excessivo ou gerador de sobrecarga ao indivíduo, a avaliação subjetiva se faz relevante enquanto elemento disparador de esforços para a sua adaptação.

$\mathrm{Na}$ análise qualitativa dos dados propiciada pela indicação dos fatores atribuídos à vivência percebida de estresse, pôde-se observar que aqueles que se consideram sob algum grau de estresse atribuem-no quase exclusivamente a fatores laborais. Salienta-se o viés de que a presente pesquisa avalia a questão da interface estresse-trabalho. Além disso, os demais itens do Roteiro Complementar avaliavam aspectos ligados ao contexto laboral, o que pode ter favorecido aos participantes limitarem-se a considerar estressores ocupacionais. No entanto, vale ressaltar que a questão das situações estressoras era aberta, permitindo a referência a fatores que não o trabalho. Destaca-se que poucos dos participantes incluíram fatores pessoais como influenciadores nas manifestações de estresse $(n=2)$, o que aponta para a centralidade do trabalho na determinação do estresse segundo os profissionais participantes.

$\mathrm{O}$ aspecto mais citado como fonte do estresse percebido esteve associado às "condições de trabalho", fazendo referência à "sobrecarga de trabalho" e à "gestão do trabalho". A sobrecarga de trabalho foi o fator mais fortemente associado à percepção de estresse pelos profissionais participantes, derivada da percepção de falta de trabalhadores e do aumento da demanda pelo serviço devido ao crescente número de usuários que procuram atendimento nas referidas instituições. A falta de investimento em recursos humanos na área da saúde mental vem sendo apontada como fator de sobrecarga e estresse da equipe, tanto pela insuficiência de profissionais (De Marco, Cítero, Moraes \& Nogueira-Martins, 2008) quanto pela falta de profissionais qualificados para o desempenho de seu trabalho (Cordeiro, 2001). A demanda por serviços de saúde mental também atua como estressor, ao gerar pressão na forma de cobrança por atendimento realizado (Souza, Vilar, Rocha, Uchoa \& Rocha, 2008).
Quanto à "gestão do trabalho", aparecem questões como estrutura física precária, dificuldades no gerenciamento administrativo, falta de suporte ao profissional, pressão no trabalho e desorganização do serviço. Aspectos relacionados à gestão e estrutura organizacionais vêm sendo associados ao estresse (Burrows \& McGrath, 2000; Irving, Dobkin \& Park, 2009). Uma vez que se situam no âmbito da organização do trabalho, apresentam-se como aspectos fora do controle dos trabalhadores, que não possuem autonomia para modificá-las.

A falta de recursos no trabalho, sejam estes de pessoal, materiais ou físicos, associada à percepção de condições precárias de atendimento, constitui um fator estressante importante para estes profissionais, uma vez que pode limitar a atuação destes, influindo diretamente na qualidade do serviço. Hannigan, Edwards, Coyle, Fothergill e Burnard (2000) verificaram que a carência de apoio institucional constitui um preditor para o esgotamento emocional. Atuar sobre os aspetos da organização e da gestão do trabalho é de fundamental importância para a redução do estresse e do esgotamento emocional (Maslach \& Leiter, 1999).

As condições de trabalho têm sido frequentemente apontadas como fatores de grande potencial estressor (Burrows \& McGrath, 2000; Macedo, 2005). Na pesquisa realizada por Rebouças, Legaly e Abelha (2007) com trabalhadores de saúde mental, precárias condições de trabalho - como instalações físicas inadequadas e falta de recursos humanos e materiais constituíram os fatores que mais contribuíram para a menor satisfação com o trabalho e maior impacto sobre a saúde do trabalhador.

Os fatores "trabalho" e "ambiente e condições de trabalho" foram considerados como muito estressantes pelos maiores percentuais de participantes $(n=15$ e $\mathrm{n}=12$, respectivamente). Considerando-se os dados da questão aberta em conjunto com os dados descritivos, depreende-se que os profissionais, em seu trabalho, significam algumas condições como estressoras, sendo estas prioritariamente relacionadas às condições de trabalho, à sua natureza e à configuração multiprofissional de trabalho, que podem ser reconhecidas como parte da proposta do atual modelo de atenção psicossocial. Irving et al. (2009) apontam a transição no contexto da saúde como associada a aumento do estresse entre os profissionais. Infere-se que, uma vez que os profissionais participantes avaliam as referidas condições como muito estressantes, podem estar vivenciando dificuldades em seu trabalho em saúde mental, especificamente no contexto da reforma psiquiátrica. Pode-se considerar 
que a formação do trabalhador influencie, uma vez que esta se embasa em uma lógica assistencial individualista voltada à cura, posturas estas problematizadas pela proposta psicossocial de atenção. Silva e Costa (2008) apontam o despreparo dos profissionais, cuja formação, pautada no modelo biomédico, não os atualiza e prepara para as novas possibilidades de intervenção na atenção em saúde.

Pela análise qualitativa da questão aberta, o segundo fator de estresse mais citado constituiu a categoria "relacionamento no trabalho". Os participantes citam a dificuldade no relacionamento interpessoal entre os profissionais como conflituoso e assinalam, em menor frequência, as dificuldades no relacionamento com os usuários. Em relação à dificuldade na execução do trabalho em equipe, observa-se que muitos profissionais apontaram a falta de confiança no colega, diferenças de valores, atribuição de valores negativos aos outros membros da equipe e depreciação de sua conduta, evidenciando a dificuldade em se trabalhar em um esquema de coordenação de atividades na qual se considera o trabalho partilhado por todos os integrantes da equipe.

As dificuldades de relacionamento na equipe afetam diretamente o seu funcionamento, fazendo com que seus membros percam o espaço para o compartilhamento de experiências no ambiente de trabalho e a oportunidade de contribuir com ideias que favoreçam a adequação às suas concepções éticas e seus próprios princípios, minando a força do grupo de trabalho como agente potencializador do suporte social, ao menos enquanto uma equipe multiprofissional formalizada. A questão do trabalho multidisciplinar e a falta de uma boa relação entre seus membros apontam para as dificuldades vivenciadas por estes profissionais quanto a esta questão.

Campos (1992) assinala que as equipes multidisciplinares em saúde mental carecem de articulação, com pouca disponibilidade para a convergência de objetivos, complementaridade e cooperação. Para o aprimoramento do trabalho em equipe, a autora alerta para a necessidade de espaços para a reflexão sobre a prática, com espaço para atividades que favoreçam até mesmo compreensão da dinâmica interna da equipe, favorecendo o seu desenvolvimento.

O fator "trabalho em equipe" foi avaliado como muito estressante por $40,0 \%$ dos profissionais na questão fechada, enquanto $36,0 \%$ o consideraram pouco estressante. Já fatores do contexto social do trabalho como "contato com os colegas" e "contato com o usuário" foram mais frequentemente avaliados como pouco estressantes $(n=12$ e $n=11$, respectivamente). Pela distribuição dos resultados, observa-se diversidade nesta avaliação, podendo-se dizer que praticamente são avaliados ao mesmo tempo como estressores e pouco estressantes.

Avaliando-se os dados descritivos com os qualitativos, pode-se apontar que a dificuldade no relacionamento centra-se na questão do trabalho conjunto e na avaliação negativa quanto à conduta profissional. Pode-se inferir, então, que a existência de conflitos na equipe multidisciplinar não parece minar a potência dos profissionais enquanto rede de apoio, ao menos para uma parcela dos participantes, uma vez que a configuração da equipe é mais frequentemente apontada como estressante, e não o contato informal com seus membros. Estudos demonstram a importância dos colegas de trabalho como recursos de apoio inclusive em interações informais (Reid et al., 1999), estando associada à redução de impacto no trabalho (Rebouças et al., 2007).

Em relação ao contato com o usuário, Pereira e Fávero (2001) verificaram que o cuidado aos usuários, no trabalho de enfermeiros, é recompensador para os profissionais, permitindo-lhes encontrar respostas de atendimento às suas aspirações. Pelo indício de que os fatores "contato com os colegas" e "contato com usuários" são frequentemente descritos na literatura como fatores de satisfação ocupacional (Rebouças et al., 2007; Pereira e Fávero, 2001), pode-se supor que o trabalho ofereça possibilidade de estabelecimento de outras relações para os profissionais, podendo consistir em um contexto social de apoio e interação entre os diferentes membros.

Dadas as dificuldades apontadas no tocante ao relacionamento no trabalho, pode-se refletir acerca do papel da instituição como mediadora da relação do profissional e seu trabalho. Podem ser formados, no ambiente de trabalho, grupos informais cujos participantes, a despeito de qualquer organização hierárquica que a instituição possa ter, podem escolher com quem se relacionar de forma mais próxima, compartilhando experiências. Assim, podem escolher de quem receber apoio e a quem recorrer, constituindo-se o grupo como fonte de satisfação das necessidades sociais do trabalho. Entretanto, na presente pesquisa, as situações formais de trocas profissionais nas discussões da equipe multiprofissional são avaliadas como estressantes. Pode-se pensar que a configuração multiprofissional portanto, formal - que é dada e, de certo modo, exigida, não se mostrou suficiente para ajudar os profissionais em suas necessidades.

Ramminger (2005) verificou que, na carência de uma equipe ideal, os profissionais partem para ações 
individuais como fonte de suporte e compartilhamento das dificuldades vivenciadas, tais como a procura e escolha de um colega com quem dividir suas angústias. Outra estratégia utilizada é a psicoterapia pessoal, a qual aparece como central para o profissional e uma iniciativa a se incentivar como ação de suporte ao profissional.

Uma estratégia suplementar que poderia favorecer uma melhor utilização dos espaços formais de trocas nos serviços seria a implementação de ações voltadas a supervisões que abarcassem o aspecto emocional do profissional. Outra poderia ser a discussão dos casos atendidos, como meio de ensinar o profissional a lidar com situações de sobrecarga no trabalho. Segundo Reid et al. (1999), a supervisão constitui um fator de proteção contra o burnout. Assim, além das questões técnicas do serviço, necessita-se de espaço para discussões sobre o próprio trabalho, o estresse dos profissionais, as dificuldades interpessoais e as estratégias compartilhadas para o enfrentamento das situações estressoras.

As iniciativas de cuidado ao trabalhador, tais como formação de equipe e supervisões, são consideradas centrais na experiência do profissional de saúde mental; já a falta de integração entre a equipe e espaços destinados à escuta e reflexão é avaliada como fator que contribui para o adoecimento do profissional (Ramminger, 2005).

$\mathrm{Na}$ presente pesquisa, os participantes apontaram como fator de estresse pessoal e associado ao trabalho a ausência de suporte ao profissional, indicando a falta de estratégias como supervisão ou psicoterapia. Isto significa que o investimento nesta forma de suporte constituiria uma estratégia organizacional e individual voltada à saúde do trabalhador.

A este respeito, cabe destacar a categoria "falta de recompensa no trabalho", em que se identificam a baixa remuneração, frustração quanto aos resultados do trabalho, falta de reconhecimento e baixa qualidade do cuidado como fatores de estresse pessoal. Tais aspectos, associados à carência de recompensas, são frequentemente relacionados ao estresse no trabalho de profissionais de saúde mental (Macedo, 2005; Gigantesco, Picardi, Chiaia, Balbi \& Morosini, 2003). A percepção de falta de um sistema de recompensa intrínseco e extrínseco ao trabalho pode resultar em um fator de desmotivação e acomodação ante as tarefas a serem desempenhadas.

Entre os fatores elencados na geração do sentimento de frustração no e pelo trabalho, os profissionais citaram a percepção de baixa resolutividade, entendida como ausência de cura e tendência à cronificação do usuário, fator que pode constituir-se como um importante gerador de insatisfação entre eles. No modo de atenção psicossocial, no entanto, busca-se uma atitude transformadora diante da doença mental e modelos de atenção em que haja disponibilidade em relação ao outro e ao diferente, numa postura de acolhimento, escuta e reflexão. Assim, observa-se que, diante das dificuldades do trabalho, empreende-se um movimento de retomada de saberes e práticas anteriores não consoantes com as práticas psicossociais de atenção, como um mecanismo defensivo (Matta, 2007). Segundo Ramminger (2008), estudos apontam que o modelo biomédico assegurava um conjunto de práticas em saúde mental cujas mudanças evidenciam as fragilidades do modo de atenção atual, em um momento de transição em que ambos os paradigmas coexistem e se contrapõem.

Conforme pontua Ishara (2007), o contato com usuários em sofrimento mental evidencia a necessidade de desenvolver competências profissionais para enfrentar os desafios da prática clínica direcionadas à construção de condições e disponibilidades internas, a fim de propiciar trocas afetivas, aprofundamento e utilização dos vínculos. Esta percepção de falta de resolutividade pode contribuir para a acomodação e a uma menor mobilização diante de situações adversas.

\section{CONSIDERAÇÕES FINAIS}

O desenvolvimento deste trabalho apontou a percepção de vivência de estresse por parte dos profissionais de saúde mental participantes do presente estudo, identificando o trabalho como central para esta vivência.

Ressalta-se a percepção dos profissionais de que o contato, tanto com os colegas quanto com os usuários, é pouco estressante para eles, o que pode favorecer o atendimento das necessidades sociais no contexto do trabalho e oferecer possibilidades de inserção e de ampliação dos vínculos sociais. No entanto, é no contexto de equipe multiprofissional que se expressam mais frequentemente as dificuldades, indicando a necessidade de intervenção com vista a minorar tais conflitos.

Os estressores do trabalho apontados associam-se a características presentes e exigidas pelo modo psicossocial de atenção decorrente da Reforma Psiquiátrica, tais como a configuração multidisciplinar da equipe e as condições de trabalho existentes. Podese inferir que os trabalhadores apresentam dificuldades em relação ao trabalho em saúde mental, 
fator capaz de influenciar negativamente a qualidade da assistência prestada aos usuários.

A avaliação sistemática dos profissionais de saúde mental pode contribuir para o estabelecimento de estratégias de cuidado ao cuidador voltadas à prevenção e minimização da ocorrência de situação estressante, o que favorece a mudança de modelo de atenção em saúde mental.

A observação do funcionamento da equipe multidisciplinar a partir do acompanhamento de reuniões administrativas e de discussão de casos pela equipe pode auxiliar na identificação de alternativas funcionais para que estes encontros se tornem possibilidades reais de troca e suporte profissional e social.

Vale ressaltar que este estudo se centrou na questão do estresse ocupacional, entretanto destaca-se que características pessoais/individuais são importantes para o desenvolvimento do estresse. Neste sentido, sugere-se que futuros estudos focalizem a verificação de ocorrência de tais características, o que pode favorecer o desenvolvimento de estratégias de controle de estresse a nível pessoal.

Especificamente no contexto de cuidado ao cuidador de usuários com sofrimento mental, novos estudos se fazem necessários com vista a auxiliar o profissional no desenvolvimento de estratégias de enfrentamento mais adaptativas às situações laborais vivenciadas como estressoras, tanto do ponto de vista individual quanto do organizacional.

Especificamente no contexto de cuidado ao cuidador de usuários com sofrimento mental, novos estudos se fazem necessários com vista a auxiliar o profissional no desenvolvimento de estratégias de enfrentamento mais adaptativas às situações laborais vivenciadas como estressoras, tanto do ponto de vista individual quanto do organizacional.

\section{REFERÊNCIAS}

Araújo, T. M., Graça, C. C. \& Araújo, E. (2003). Estresse ocupacional e saúde: contribuições do modelo demanda - controle. Ciência e Saúde Coletiva, 8(4), 991-1003.

Bardin, L. (1979). Análise de conteúdo. Lisboa: Edições 70.

Burrows, D. \& McGrath, C. (2000). Stress and mental health professionals. Stress Medicine, 16, 269-270.

Campos, M. A. (1992). O trabalho em equipe multiprofissional: uma reflexão crítica. Jornal Brasileiro Psiquiatria, 41(6), 255-257.

Carson, J. \& Fagin, L. (1996). Stress in mental health professionals: a cause for concern or an inevitable part of the job. International Journal of Social Psychiatry, 42(2), 79-81.

Cordeiro, H. (2001). Descentralização, universalidade e equidade nas reformas da saúde. Cien. Saúde Coletiva 6(2), 319-28.
De Marco, P. F., Cítero, V. A., Moraes, E. \& Nogueira-Martins, L. A. (2008). O impacto do trabalho em saúde mental: transtornos psiquiátricos menores, qualidade de vida e satisfação profissional. Jornal Brasileiro de Psiquiatria, 57(3), 178-83.

Gigantesco, A., Picardi, A., Chiaia, E., Balbi, A. \& Morosini, P. (2003). Job satisfaction among mental health professionals in Rome, Italy. J. Community Ment. Health, 39(4), 349-355.

Hannigan, B., Edwards, D., Coyle, D., Fothergill, A. \& Burnard, P. (2000). Burnout in community mental health nurses: findings from the all-Wales stress study. Journal Psychiatry Mental Health Nursing, 7(2), 127-134.

Irving, J. A., Dobkin, P. L. \& Park, J. (2009). Cultivating mindfulness in health care professionals: A review of empirical studies of mindfulness-based stress reduction (MBSR). Complementary Therapies in Clinical Practice, 15(2), 61-66.

Ishara, S. (2007). Equipes de saúde mental: avaliação da satisfação e do impacto do trabalho em hospitalização integral e parcial. Tese de Doutorado, Faculdade de Medicina de Ribeirão Preto da USP, Ribeirão Preto.

Lazarus, R. S. \& Folkman, S. (1984). Stress, appraisal and coping. New York: Sringer Publishing Company.

Lipp, M. E. N. \& Malagris, L. N. (1998). Manejo de estresse. Em B. Range (Ed.), Psicoterapia Comportamental e Cognitiva (pp. 279292). São Paulo: Editorial Psy.

Lipp, M. E. N. (2000). Manual do Inventário de Sintomas de Stress para Adultos de Lipp. São Paulo: Casa do Psicólogo.

Macedo, Z. D. (2005). Dificuldades que afetam os profissionais da saúde mental na rede pública da região oeste de Santa Catarina. Dissertação de Mestrado, Universidade do Oeste de Santa Catarina, Joaçaba.

Maslach, C. \& Leiter, M. P. (1999). Trabalho: Fonte de prazer ou desgaste? Campinas: Papirus editora.

Maslach, C., Schaufeli, W. B. \& Leiter, M. P. (2001). Job Burnout. Annual Review Psychology, 52, 397-422.

Matta, P. H. D. (2007). O trabalho em saúde mental: acolhendo a crise do cuidador. Trabalho de Conclusão de Curso, Faculdade de Ciências Médicas da UNICAMP, Campinas.

Paschoal, T. \& Tamayo, A. (2004). Validação da escala de estresse no trabalho. Estud. Psicol. (Natal), 9(1), 45-52.

Pereira, M. C. A. \& Fávero, N. (2001). A motivação no trabalho da equipe de enfermagem. Revista Latino-americana de Enfermagem, 9(4), 7-12.

Ramminger, T. (2005). Trabalhadores de saúde mental: reforma psiquiátrica, saúde do trabalhador e modos de subjetivação nos serviços de saúde mental. Dissertação de Mestrado, Instituto de Psicologia da Universidade Federal do Rio Grande do Sul, Porto Alegre.

Ramminger, T. (2008). Saúde do trabalhador de Saúde Mental: uma revisão dos estudos brasileiros. Saúde debate; 32(78-80),60-71.

Rebouças, D., Legay, L. F. \& Abelha, L. (2007). Satisfação com o trabalho e impacto causado nos profissionais de serviço de saúde mental. Revista de Saúde Pública, 41(2), 244-250.

Reid, Y, Johnson, S., Morant, N., Kuipers, E., Szmukle, G., Bebbington, P., Thornicroft, G. \& Prosser, D. (1999). Improving support for mental health staff: a qualitative study. Social Psychiatry and Psychiatric Epidemiology, 34(6), 309-315.

Selye, H. (1956). The stress of life. New York: Longmans. 
Silva, E. A. \& Costa, I.I. (2008). Saúde mental dos trabalhadores em saúde mental: estudo exploratório com os profissionais dos Centros de Atenção Psicossocial de Goiânia/GO. Psicologia Rev, 14(1), 83-106.

Souza, E. C. F., Vilar, R. L. A., Rocha, N. S. D., Uchoa, A. C. \& Rocha, P. M. (2008). Acesso e acolhimento na atenção básica: uma análise da percepção dos usuários e profissionais de saúde. Cad. Saúde Pública, 24(Sup1), S100-S110.

Stacciarini, J. M. \& Troccolo, B. T. (2001). O estresse na atividade ocupacional do enfermeiro. Rev Latino-am Enfermagem, 9(2), 17-25.

Swoboda, H, Sibitz, I., Fruhwald, S., Klug, G., Bauer, B. \& Priebe, S. (2006). How do community-based mental health staff in Austria perceive their job? - a qualitative study. Psychiatric Prax, 32(8), 386-392.

Yarker, J., Donaldson-Feilder, E. \& Flaxman, P. (2007). Management competencies for preventing and reducing stress at work: Identifying and developing the management behaviours necessary to implement the HSE Management Standards. Health and Safety Executive Books. Recuperado em 22 de outubro de 2009 de http://www.hse.gov.uk/research/rrpdf/rr553.pdf

Recebido em 25/09/2008

Aceito em 18/11/2009

\section{Endereço para correspondência:}

Carmen Lúcia Cardoso, Departamento de Psicologia e Educação. Faculdade de Filosofia, Ciências e Letras de Ribeirão Preto. Universidade de São Paulo. Av: Bandeirantes, 3900, CEP 14040-901, Ribeirão Preto-SP, Brasil. E-mail: carmen@ ffclrp.usp.br. 